\title{
Reservations and Complexity: The Relationship between Politeness and Emotional Intelligence
}

\author{
Masoumeh Ahmadi Shirazi \\ University of Tehran
}

\begin{abstract}
.
Although politeness has a major place in many languages, and should be noticeable in everyday behavior, many people, especially the youth, in the current generation do not always act politely. Politeness is not considered as much as it used to be. This may be attributed to individuals' low Emotional Intelligence (EI). The present study aimed to investigate the relationship between EQ and politeness. To this end, a group of 50 EFL Iranian learners participated in this study by responding to politeness and EI Appraisal Tests. The first test measured politeness suggested by Brown and Levinson (1978), which had 25 items with four possible responses whose scores ranged between 1 (the least polite) and 4 (the politest). The EQ questionnaire was a self-report appraisal test with 28 items measuring EI through 5-point Likert Scale. The correlational analysis revealed there was not a statistically significant relationship between EQ and politeness of EFL learners. The second purpose of the study was to investigate the relationship between politeness and the four skills of EQ. The results revealed that politeness does not have any statisically significant relationship with any of the four EQ skills (i.e., Self-Awareness, Self-Management, Social Awareness, and Relationship Management) among EFL Iranian learners. These findings suggest that politeness is a complicated phenomenon as is the case with EQ requiring further investigation.
\end{abstract}

Keywords: personal competence, social competence, emotional management, Emotional Quotient 


\section{Introduction}

In today's fast-paced world of competitive workplaces and chaotic personal lives, each of us is searching for effective tools that can make our schedules, behaviors, and relationships more manageable.

Additionally, some people in unusual circumstances do not speak or act wisely. When they argue, reject an opinion or oppose something, they react according to their feelings and emotions. They do not behave politely. As a result, they face some consequences.

Emotional competencies are found to be the integral parts of everyday life, promoting the study of emotional quotient (EQ) by researchers from various disciplines (Cote \& Miners, 2006). The relationship of EI with some organizationally relevant outcome variables such as self-rated performance, self-efficacy, job satisfaction, personal effectiveness, general health, turnover intention, commitment, trust and organizational effectiveness was investigated by previous studies (Jain, 2011; Jain \& Sinha, 2005; Sinha \& Jain, 2004). Moreover, other recent empirical studies found that EI is as an important tool associated with positive outcomes such as prosocial behavior, parental warmth, and positive family and peer relations (Dulewicz, Higgs \& Slaski, 2003; Salovey \& Mayer,1990; Mayer, Caruso, \& Salovey, 1999; Rice, 1999).

The underlying assumption of all these studies is that EI is a powerful tool for people to enhance their intrapersonal and interpersonal effectiveness both at work and home. EI provides the potential for performance, rather than performance itself; how one would use this potential is a matter of personal choice. In other words, EI skills can be used not only to manage one's performance and relationships with others, but also to manage one's impression on supervisor and manipulate others at work place. Thus, it can be a powerful tool for leading business leaders and managers as well as for politicians.

Everyday conversation will help people to get whatever they are interested in. When they are with a group of friends, they can say to them, "Go get me that plate!" or "Shut-up!" However, when we are surrounded by a group of adults at a formal occasion in which our parents attend, we must say, "Could you please pass me that plate, if you don't mind?", and "I'm sorry, I don't mean to interrupt, but I am not able to hear the speaker in the front of the room." In different social situations, we are obliged to adjust our use of language to fit the occasion. It would seem socially unacceptable if you were to speak the same way to adults as you would to your friends.

In today's modern world, much interaction takes place at both personal and professional levels. One has to deal with all kinds of people with different temperaments. A person who can get along well with others is more successful in life. For this reason one has to be polite. In this regard, politeness can help individuals to have better communication skills.

In order to be successful and fulfilled nowadays, we must learn to maximize our EQ skills. Everyone employs a unique blend of reason and feeling to achieve the greatest results (Bradberry \& Greaves, 2009). Additionally, in order to be successful at work, at home and in business, we must improve our communication skills. 
Though being impolite does not have any defined penalty, it has some consequences. You may lose your job, people who are important to you, get isolated and hurt and so on. Social problems related to politeness are increasing day by day.

Nowadays, the importance of both politeness and EQ in order to succeed in social, professional, and family life should be observed. Therefore, this study aims to analyze the relationship between these variables. We would like to know whether people with higher EQ are more polite and vice versa.

The purpose of this study is to investigate whether there is a relationship between EQ and Politeness. If a relationship between EQ and politeness exists, it is possible to increase the degree of politeness by means of improving EQ. EQ alters over time. The rate is not high. It does not change dramatically naturally. The rate is low unless you improve it by practicing. If you do not practice to raise your EQ, it changes but it takes about ten years for a significant change You can increase your EQ in a couple of months by practicing and working on (Bradberry \& Greaves, 2009). The current study was designed to address the following research question:

1. Is there any relationship between politeness and emotional intelligence?

2. Is there any relationship between politeness and EQ skills including self-awareness, selfmanagement, social awareness, and relationship management?

\section{Methods}

\subsection{Participants}

In this study the participants were Iranian students. The students of Islamic Azad University, South branch were chosen as the statistical population of this study. Sampling method used in this study was random replacement (Delavar, 2011).

In this study 50 Iranian students from the mentioned statistical population were chosen as the samples. In terms of gender, $9(18 \%)$ were male and $41(82 \%)$ were female. In terms of age, out of 50 participants, 5 (10\%) were 18-20 years of age, 27 (54\%) were between 20 and $25,9(18 \%)$ were $25-30$ years of age, and $9(18 \%)$ were between 30 and 35 . In terms of degree $45(90 \%)$ had associate degrees, $3(6 \%)$ had bachelor degrees and $2(4 \%)$ had master's degrees. They were all chosen from EFL students who had EFL degrees (Table 1).

\subsection{Materials and Instruments}

The first instrument of the study is established on the theory of Brown and Levinson. Brown and Levinson's politeness theory was developed in 1978. It includes five strategies (baldly, positive politeness, negative politeness, off record and no Face threatening Act). The test had twenty five multiple choice questions with 4 probable responses. For responses a, b, c and $\mathrm{d}, 1,2,3$ and 4 points were considered respectively. The total scores were between 0 and 100. Higher scores were the indication of higher politeness. 
The second test is called EQ appraisal test and is established on Goleman's theory in 1996. This test had twenty eight multiple choice questions with 5 probable responses. The responses were never, rarely, sometimes, usually, almost always and always. The test measured 4 EQ skills that are self-awareness, self-management, social awareness and social management. Question number one to six measured self-awareness, number seven to fifteen measure selfmanagement, sixteen to twenty measured social awareness and twenty one to twenty eight measured relationship management. The maximum score for each EQ skill was 100. The four skills of EQ scores were averaged out. The scores were between 0 and 100. Higher scores were the indication of higher EQ.

\section{Results}

Fifty questionnaires were chosen for data analysis. Regarding the research questions, in order to find any relationship between EQ and politeness, and also politeness and skills of EQ (Self-awareness, Self-management, Social awareness, Relationship management) Pearson product moment correlation coefficient was performed. The procedure and results of this analysis are now presented.

\subsection{Politeness and EQ}

The relationship between Politeness and EQ was investigated using Pearson product moment correlation coefficient. There was not a correlation between the two variables, $(\mathrm{r}=-$ $0.118, \mathrm{n}=50, \mathrm{p}=0.416)$. Since the $\mathrm{p}$-value calculated $(\mathrm{p}=0.416)$ was greater than the significance level (0.05), the null hypothesis cannot be rejected.

\subsection{Politeness and Self-awareness}

There was not a correlation between the two variables, $(\mathrm{r}=-0.150, \mathrm{n}=50, \mathrm{p}=0.300)$. Since the $\mathrm{p}$-value calculated $(\mathrm{p}=0.300)$ was greater than the significance level $(0.05)$, the null hypothesis cannot be rejected.

\subsection{Politeness and Self-management}

There was not a correlation between the two variables, $(r=-0.253, n=50, p=0.076)$. Since the $\mathrm{p}$-value calculated $(\mathrm{p}=0.076)$ was greater than the significance level $(0.05)$, the null hypothesis cannot be rejected.

\subsection{Politeness and Social-awareness}

There was not a correlation between the two variables, $(r=0.003, n=50, p=0.986)$. Since the $\mathrm{p}$-value calculated $(\mathrm{p}=0.986)$ was greater than the significance level $(0.05)$, the null hypothesis cannot be rejected.

\subsection{Politeness and Relationship management}

There was not a correlation between the two variables, $(r=0.038, n=50, p=0.794)$. Since the $\mathrm{p}$-value calculated $(\mathrm{p}=0.794)$ was greater than the significance level $(0.05)$, the null hypothesis cannot be rejected. 


\section{Discussion}

Accoridng to the findings of the study, none of our hunches came true. Although finding no correlations between politeness and EI is not surprising due to the complexity of both, it can be detrimental to the culture of many societies. Politeness issue changes from person to person, context to context, culture to culture, youth to elderly and poor to rich. There are many a variable that the researchers cannot control so as to measure such intricate concepts. Even their measurement brings with itself many doubts and hesitations, especially with the increasing interest in researching the differences among individuals and different personality types. As was observed, all null hypotheses of the research could not be rejected, hence no relation between politeness and EI and its components.

\section{Conclusion}

A group of Iranian male and female EFL learners who were between the ages of 18-35 were asked to sit for the tests. Although a positive correlation was expected, the analysis of collected data indicates that there is no correlation between EQ and politeness. Since the result of the analysis was unexpected, the process of data collection was redone. The second time, the tests were administered to another group. The result was the same as what we obtained through the first attempt. In this study two different analyses were considered. In the first analysis all the data were considered and in the second analysis the outliers were initially excluded. According to the findings, the null hypothesis which states that there is no relationship between emotional intelligence and politeness was not rejected. Moreover, no relationship was found between politeness and skills of EQ (Self-awareness, Selfmanagement, Social awareness, Relationship management).

Due to the result of the study, EQ and politeness are independent. Since there was no relationship between politeness and EQ, no suggestion can be made about different strategies to improve politeness by means of improving EQ or vice versa. If one wishes to improve the politeness, other strategies should be adopted such as practicing the ability to communicate appropriately at school. Given that schools are the best environment to practice the socially correct manners and behaviors, they are the best place to practice conversation as an integral part of these manners. An effective conversational behavior is the one accompanied by politeness, as impoliteness exhibits negative feelings towards the hearer, and may hurt the hearer's feelings or make the hearer angry. Same concept applies to EQ as well. Students should learn EQ related skills during their studies so that when they graduate and become the active part of the society, they have already developed politeness and EQ skills.

Knowing that EQ and politeness in this study has no relationship which violates what we thought of at the outset of the study, does not lessen the importance of neither EQ nor politeness. We are able to declare our findings to other groups to construct a different method of teaching, or another type of testing, a new framework for developing textbooks the basis of which should not disregard these two issues of our concern. Teachers can practice politeness in their dialog with their students in the classroom while they are totally aware of the role EQ plays in changing the way people start talking to one another. Testers, also, can include these two issues in their tests; it is not confined to speaking test but it can be practiced for reading 
3rdInternational Conference on Applied Research in

EDUCATION

04-06 June,2021

Barcelona, Spain

test as well. Especially, those involved in the study of discourse in general, and critical discourse analysis in particular need to be concerned about both EQ and politeness since the incorporation of these issues in tests like CDT or CCDT has the potential to change the results previously obtained without considering these two issues.

\section{References}

Bradberry, T. \& Greaves, J. (2009). Emotional Intelligence 2.0. Publications of Talentsmart, San Diego: Test of Emotional Intelligence appraisal.

Dulewicz, V., Higgs, M., \& Slaski. M. (2003). Measuring emotional intelligence: Content, construct and criterion-related validity. Journal of Managerial Psychology, vol.18, pp. 405-420.

Jain, A.K. (2009). Exploring the relative relevance of organizational citizenship behavior and Emotional Intelligence. Journal of the Indian Academy of Applied Psychology, vol. 35, pp. 87-97.

Jain, A. K., \& Sinha, A. K. (2005). General health in organizations: Relative relevance of emotional intelligence, trust and organizational support. International Journal of Stress Management, vol.12, pp. 257-273.

Mayer, J. D., Caruso. \& Salovey, P. (1999). Emotional Intelligence meets traditional standards for an intelligence. Intelligence, vol. 27, pp. 267-298.

Rice, P. L. (1999). Stress and Health. Pacific Grove, CA: Brooks/ Cole Publishing.

Salovey, P. \& Mayer, J. D. (1990). Emotional intelligence. Imagination, Cognition, and Personality, vol. 9, pp. 185-211.

Sinha, A. K., \& Jain, A, K. (2004). Emotional Intelligence: Imperatives for organizationally relevant outcome variables. Psychological Studies, vol. 49, pp. 81-96. 